\title{
Training Medical Communication Skills with Virtual Patients: Literature Review and Directions for Future Research
}

\author{
Edoardo Battegazzorre ${ }^{[0000-0002-1383-3285]}$, Andrea \\ Bottino $^{[0000-0002-8894-5089]}$, and Fabrizio Lamberti ${ }^{[0000-0001-7703-1372]}$ \\ DAUIN, Politecnico di Torino, Corso Duca degli Abruzzi 24, 10143 Torino, Italy \\ \{edoardo.battegazzorre, andrea.bottino, fabrizio.lamberti\}@polito.it
}

\begin{abstract}
Effective communication is a crucial skill for healthcare providers since it leads to better patient health, satisfaction and avoids malpractice claims. In standard medical education, students' communication skills are trained with role-playing and Standardized Patients (SPs), i.e., actors. However, SPs are difficult to standardize, and are very resource consuming. Virtual Patients (VPs) are interactive computer-based systems that represent a valuable alternative to SPs. VPs are capable of portraying patients in realistic clinical scenarios and engage learners in realistic conversations. Approaching medical communication skill training with VPs has been an active research area in the last ten years. As a result, the number of works in this field has grown significantly. The objective of this work is to survey the recent literature, assessing the state of the art of this technology with a specific focus on the instructional and technical design of VP simulations. After having classified and analysed the VPs selected for our research, we identified several areas that require further investigation, and we drafted practical recommendations for VP developers on design aspects that, based on our findings, are pivotal to create novel and effective VP simulations or improve existing ones.
\end{abstract}

Keywords: Virtual Patient · Embodied Conversational Agent · ProviderPatient Communication · Instructional Design · Technical Design

\section{Introduction}

The communication between patients and doctors is a central component of health care practice. On the one hand, good doctor-patient communication help physicians better identify patient's needs, perceptions and expectations 22. On the other hand, it is not surprising that patients rate open communication as one of the most important aspects of their relationship with the physicians [12]. Research has shown that an effective, patient-centered communication is important to increase patient satisfaction [17/20|24/34|39|49|61]. It can have also beneficial effects on patient's health, improving physiologic measures as blood pressure and glucose levels [62, increasing understanding and adherence to therapy [36, and even creating a placebo effect in some cases 35. Conversely, a poor 
grasp of communication skills can be detrimental to both the patient's and their relatives' health 33 34, and may lead to malpractice accusations.

Communication is a complex phenomenon that's not restricted to the verbal domain. As outlined in [34, there are several critical aspects that have been subject to patients' complaints. They include elements of non-verbal communication (e.g., lack of eye-contact, negative facial expressions, use of "improper" prosodic features), inappropriate choice of words, lack of pauses to let patients ask questions, lack of listening, issues with the information given, and lack of empathy or even disrespect and poor attitudes.

Given the relevance of the problem, a primary goal in healthcare is training clinicians and healthcare providers in developing effective communication skills. Today, standardized patients (SP, i.e., actors who are instructed to represent a patient during a clinical encounter with a healthcare provider) are considered the gold standard in such training programs. SPs provide students with the opportunity to learn and practice both technical and non-technical skills in an environment capable of reproducing the realism of the doctor-patient relationship. These simulated environments are less stressful for the students, who are not required to interact with a real patient 1838. However, SPs are difficult to standardize, since their performance heavily depend on the actors' skills, and their recruitment and training can become very costly [46.

A practical alternative to SPs is represented by virtual patients (VPs), i.e., interactive computer simulations capable of portraying a patient in a clinical scenario in a realistic way. VPs are virtual agents that have a human appearance and the ability to respond to users and engage in communication patterns typical of a real conversation. They can be equipped with external sensors capable of capturing a wide range of non-verbal clues (user's gestures and motions, expressions and line of sight) and use them to modulate the evolution of the conversation. In the field of communication skill learning, VPs have the same advantages over SPs and are characterized by comparable learning outcomes [53. They are cost-effective solutions since they can be developed once and used many times. They can be deployed as in-class or self-learning tools that students can use at their own pace and at any place. They can also be integrated into sophisticated software platforms that include automatic learner assessment, feedback and debriefing sessions. Finally, VP simulations can present, with reasonable accuracy, difficult or rare cases with a high degree of repeatability 65. and, compared to SPs, are also easier to standardize [57].

VPs for provider-patient communication have been surveyed in several works. Bearman et al. 6] conducted a systematic review on VPs focused on developing empathy-related skills, but the Authors did not extend their analysis to communication as a whole. A more recent review [55] investigated the specific context of pharmacist-patient counselling, focusing on the development of knowledge, skills, confidence, engagement with learning, and user satisfaction. However, the work did not discuss in depth the effects of specific design choices. Another integrative review [51] focused on non-technical skills like situational awareness, decision-making, teamwork, leadership, and communication, but did not con- 
sider the technical perspective and, like the previous one, it did not elaborate on the impact of specific instructional or design features. Finally, the Authors of 41] performed a systematic review on VPs focused on communication, analyzing which features of instructional design (i.e., the definition of methods, processes and strategies that guide learners to achieve the training objectives) and technical design (i.e., the definition of the technological components aimed to support and implement the envisaged instructional design) are most effective in VP simulations. Unfortunately, the time span of the survey was limited to 2006-2018, and the number of studies remaining from the application of the inclusion and exclusion criteria was rather small (14 works, with only eight discussed in detail).

Based on the above analysis, it was our opinion that a thorough analysis of the instructional and technical design elements as well as of the technological components (sensory system, speech understanding, interaction devices, virtual reality, VR, and augmented reality, AR, etc.) and related relevant concepts (like immersion and presence) that are involved in the development of the considered learning tools was actually missing. Hence, we tried to fill this gap through the review reported in the present paper. Similarly to [41, our study is centered on the instructional and technical design of VP simulations. However, we propose a different approach to the analysis of these two components, by performing in particular a deeper investigation of the technical aspects. Then, based on our findings, we also identify current limitations and potentially unexplored areas with the aim to foster further research and developments in the field.

The rest of the paper is organized as follows. In Section 2, the literature review protocol is first introduced. Afterwards, Section 3 presents and discusses the research results. Section 4 highlights the gaps that we identified and the directions that future studies could take in order to address them. Finally, conclusions are given in Section 5 .

\section{Literature review protocol}

As illustrated in the previous section, we performed a literature review to document the current state-of-the-art of the use of VPs for medical communication skill learning and to identify possible areas where further research is needed. The purpose of this review was to understand the instructional and technical design principles and the efficacy of these elements in achieving the expected learning outcomes. To this end, we developed the following guiding questions in order to help focusing information extraction.

- RQ1: What are the latest technical developments in the field of communication-oriented VPs?

- RQ2: Which instructional and technical design features are employed most commonly in VP design?

- RQ3: Which instructional and technical VP design features are more effective for learning communication skills? And which of these features are most appreciated by the users? 
The search process, carried out mainly between March and May 2020, started with an automated approach targeting four scientific paper databases, namely Scopus, PubMed, ACM Digital Library and IEEE Xplore. For each database we performed a search based on the main and derivative keywords (virtual patient OR (serious game AND healthcare)) AND (communication), limiting the results to papers published from 2015 onward. The choice of this date was made with the aim to survey only the most recent developments in the field and avoid excessive overlaps with previous literature reviews (e.g., with [51 and [41]).

The papers found were post-processed in order to remove repeated entries and exclude reviews, editorials, abstracts, posters and panel discussions. The remaining 306 papers were analyzed by reading over their title, abstract, and introduction, and classified as either relevant or irrelevant based on the following criteria: (i) does the study relate to any of the design elements of interest (instructional or technical)? and, (ii) does the study disclose at least some of the design choices made by the Authors? If the answer to any of these questions was no, then the paper was excluded. After this step, each of the 70 accepted papers was read completely by at least one reviewer, who also assessed its quality. Its references were also analyzed according to the aforementioned screening process.

At the end of the search process, we selected a total of 28 papers. Among them, we identified a number of works that referred to the same VP, but in different experimental settings or in different phases of the development process. Since our interest was in analyzing the VP design rather than the detailed outcomes of possible experiments, papers sharing the same VP were grouped together, obtaining a total of 21 VPs (17 of them had not been discussed in previous surveys, and only four of them were in common with [41, namely Banszki 4153, CynthiaYoungVP [19], MPathic-VR [21|40], and NERVE [25|26 37]).

In order to capture the main characteristics of problems and solutions discussed in these papers, we introduced a taxonomy of terms for the instructional and technical design elements, whose initial version was defined based on the Authors' expertise. Based on intermediate findings, this taxonomy was further refined into the final one introduced in Section 3. All the Authors categorized the selected VPs according to this taxonomy, and any disagreement was solved by discussion. Finally, as a last step, we searched for references related to the open problems and potential areas of research identified during the analysis.

\section{Results and discussion}

In this section, we present the result of our research. As stated in Section 2, the selected VPs have been labeled according to the taxonomy of terms summarized in Tables 1 and 2, The definition of the identified categories (which differs to a large extent from the one presented in [41]) is introduced in the following subsections, where we also discuss the survey results relative to each group. We first introduce the instructional design elements, which are connected to the technical elements necessary to realize them; afterwards, we discuss the design choices related to the technical and technological components of the simulations. 
Table 1. Synopsis of the reviewed VPs for each instructional design category

\begin{tabular}{|c|c|c|}
\hline \multicolumn{3}{|r|}{ Instructional design } \\
\hline Category & Subcategory & Virtual Patients \\
\hline \multirow[t]{2}{*}{ Structure } & Narrative & $\begin{array}{l}\text { HOLLIE 1], AtRiskInPrimaryCare }[2, \text { Dupuy }[15, \text {, CynthiaY- } \\
\text { oungVP 19], Jacklin 28 29], MPathic-VR 21|40], Communicate! } \\
\text { |31, Marei 44, Ochs 47, Szilas 63] }\end{array}$ \\
\hline & \begin{tabular}{|lr} 
Narrative & + \\
Problem & solv- \\
ing & \\
\end{tabular} & $\begin{array}{l}\text { Banszki 4 53], NERVE } 25 \text { 26 37], Maicher [43], Suicide Preven- } \\
\text { tion [48, VSPR [50|52], Richardson [54], CESTOLVRClinic [59], } \\
\text { Schoenthaler 60], Washburn [66], UTTimePortal 6869], Zlotos } \\
\text { 70 }\end{array}$ \\
\hline \multirow[t]{3}{*}{ Unfolding } & Closed-option & 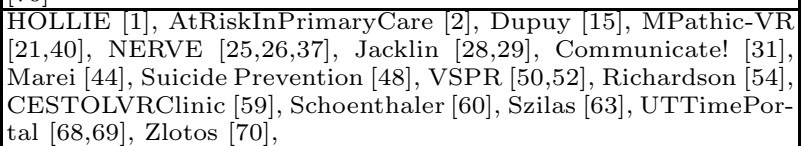 \\
\hline & Open-option & $\begin{array}{l}\text { Banszki 4 53, CynthiaYoungVP 19, NERVE 25 26137, Maicher } \\
\text { 43, Washburn 66] }\end{array}$ \\
\hline & Hybrid & Ochs [4] \\
\hline \multirow{8}{*}{ Feedback } & Replay feature & $\begin{array}{l}\text { Dupuy [15], Communicate! 31], Ochs [4], VSPR [50|52], Zlotos } \\
\text { 70 }\end{array}$ \\
\hline & $\begin{array}{l}\text { Virtual instruc- } \\
\text { tor }\end{array}$ & $\begin{array}{l}\text { At-Risk in Primary Care }[2 \text {, Suicide Prevention [48, Schoenthaler } \\
60 \text { ] }\end{array}$ \\
\hline & $\begin{array}{l}\text { Multiple ses- } \\
\text { sion structure }\end{array}$ & MPathic-VR $[2140]$ \\
\hline & $\begin{array}{l}\text { Quantitative } \\
\text { emotional } \\
\text { feedback }\end{array}$ & At-Risk in Primary Care $[2]$, Schoenthaler [60] \\
\hline & $\begin{array}{l}\text { Qualitative per- } \\
\text { sonalized post- } \\
\text { feedback }\end{array}$ & \begin{tabular}{|l} 
Jacklin $[28$ \\
29
\end{tabular} \\
\hline & $\begin{array}{l}\text { Empathy feed- } \\
\text { back }\end{array}$ & CynthiaYoungVP 19 \\
\hline & $\begin{array}{l}\text { Clinical discov- } \\
\text { eries available }\end{array}$ & 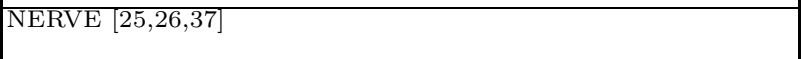 \\
\hline & Game elements & 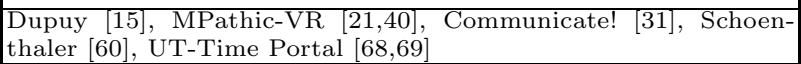 \\
\hline \multirow[t]{3}{*}{ Gamification } & Scoring system & 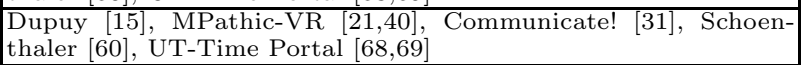 \\
\hline & Badge system & $\left.\begin{array}{|lll}\text { UTTimePortal } & 68 & 69\end{array}\right]$ \\
\hline & $\begin{array}{l}\text { Countdown } \\
\text { timed events }\end{array}$ & HOLLIE 1 \\
\hline
\end{tabular}

Finally, we discuss the experimental evidences related to the effectiveness of the identified design elements.

\subsection{Instructional design}

This category encompasses various instructional design aspects implemented in the VP scenario, such as how the VP delivers (and facilitates) learning activities and if (and how) it provides scaffolded support to improve learner's performance.

Structure. The structure defines the hierarchical organization and presentation of VP-related information within the simulation. According to [5], two non-mutually exclusive approaches (i.e., narrative and problem solving) can be defined. The narrative VPs are characterized by a coherent storyline, with a focus on cause-effect decisions that have a direct impact on the evolution of the simulation. These VPs present the patient as more than a mere collection of data and statistics, and devote a certain degree of attention to interpersonal and 
Table 2. Synopsis of the reviewed VPs for each technical design category

\begin{tabular}{|c|c|c|}
\hline & & Technical Design \\
\hline Category & Subcategory & Virtual Patients \\
\hline \multirow{5}{*}{$\begin{array}{l}\text { Presentation } \\
\text { format }\end{array}$} & Image & HOLLIE 1, Marei 44 \\
\hline & Video & CynthiaYoungVP 19 , Suicide Prevention 48 , VSPR 15052 \\
\hline & Desktop VR & 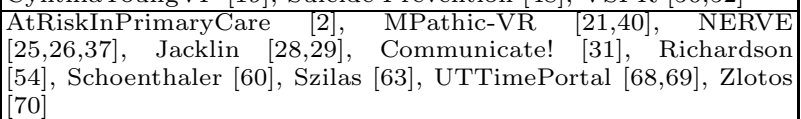 \\
\hline & $\begin{array}{ll}\text { Large } & \text { volume } \\
\text { display } & \end{array}$ & Dupuy 15, Banszki 453, Maicher 43, Washburn 66 \\
\hline & Immersive $V R$ & Ochs $[47$, CESTOLVRClinic $[59]$ \\
\hline \multirow[t]{4}{*}{$\begin{array}{l}\text { Input } \\
\text { interface }\end{array}$} & Typed & $\begin{array}{l}\text { HOLLIE 1], AtRiskInPrimaryCare 2], CynthiaYoungVP } 19] \\
\text { NERVE 25 26 37, Jacklin 28 29], Communicate! [31], Maicher } \\
\text { 43], Marei [44], Suicide Prevention [48], VSPR [50 52], Richard- } \\
\text { son 54, CESTOLVRClinic [59], Schoenthaler 60], Szilas 63], UT- } \\
\text { TimePortal 68 69, Zlotos 70] }\end{array}$ \\
\hline & $\begin{array}{l}\text { Voice- } \\
\text { controlled }\end{array}$ & $\begin{array}{l}\text { Banszki } 4 \mid 53], \text { Dupuy }[15], \text { MPathic-VR [2140], Maicher [43], } \\
\text { Ochs 47], CESTOLVRClinic [59] }\end{array}$ \\
\hline & Hybrid & Washburn 66 \\
\hline & Non-verbal & $\begin{array}{l}\text { Banszki 4/53, Dupuy 15, MPathic-VR 2140, Maicher 43, } \\
\text { CESTOLVRClinic [59] }\end{array}$ \\
\hline \multirow[t]{3}{*}{ Distribution } & Standalone & $\begin{array}{l}\text { Banszki 453], Dupuy }[15] \text {, MPathic-VR [2140], Maicher [43], } \\
\text { Marei 44], Ochs 47, CESTOLVRClinic [59], Szilas 63], Wash- } \\
\text { burn 66] }\end{array}$ \\
\hline & Web-based & 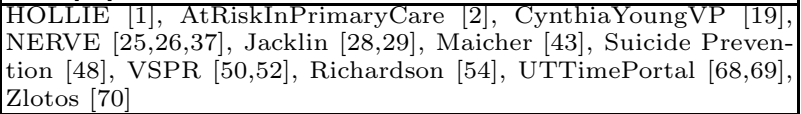 \\
\hline & Undisclosed & \begin{tabular}{|l|l|} 
Communicate! 31$],$ Schoenthaler 60$]$ \\
\end{tabular} \\
\hline
\end{tabular}

communication aspects of the provider-patient interaction. On the contrary, the problem solving VPs are mainly used to support inquiry-based learning scenarios such as teaching clinical reasoning, differential diagnosis, and history-taking skills. These contexts do not usually concern portraying authentic communicative acts, since they mainly involve making questions and observations.

Scholars and researchers recognize the power of narrative design in the creation of meaningful learning experiences [5|44]. Narrative-based simulations that reflect the consequences of the choices and the actions made by the learner can lead to the development of more effective VPs. In particular, for VPs used to teach communication skills, experimental evidence supports the value of narrative design [5]. Thus, it is not surprising that all the VPs presented in the selected works are based on this approach. Nevertheless, it is interesting to note that 10 out the 21 VPs analyzed integrate the narrative design with a problem solving component. This component aims to teach particular skills like history-taking (Cynthia Young VP [19, NERVE [25|26137, Maicher 43]), clinical reasoning (VSPR [50152, Richardson [54], Washburn [66], UT-Time Portal 68|69], Zlotos [70]), physical examinations (HOLLIE [1, NERVE [25|26137, CESTOL VR Clinic [59]), compilation and consultation of electronic medical records (HOLLIE 1), Maicher [43), and medication administration (HOLLIE 11).

Unfolding. Given the prominence of narrative design in the development of VPs for communication skill training, another relevant design element is defining how the narrative may unfold, and how the simulation can evolve between 
different states. A preliminary subdivision can be made among linear and nonlinear narratives. In the former design, VPs have a linear path to follow and the decisions, questions and options possibly presented to the learner do not influence the simulation outcome. It is clear that this design severely limits learning effectiveness, and none of the works included in this survey implemented it.

On the contrary, the non-linear navigational structure of VPs offers learners a greater flexibility, and an higher degrees of interactivity and control. In this case, two further choices are possible. In the closed-option design, the simulation advances to the next state by selecting one of the possible alternatives or explicit paths offered to learners. Simulation states are organized in a hierarchical structure (similar to that of the "choose your own adventure" books), which stresses the cause-effect relation of the user's choices. The open-option design (sometimes referred in the literature as "free-text" 28/30|45] or "open-chat" [26]) can be used to develop free-form simulations where states are organized in a partially or fully interconnected structure, and users are free to interact with the VP as they wish, thus emulating the flow of a real conversation. As we will discuss more in detail in Section 3.2. learners can formulate questions and statements by either typing or having their speech transcribed into written words using speech-to-text software. Then, the application parses the text and elaborates a proper response. The VP state progression can be influenced also by non-verbal cues such as gestures, body posture, expressions and sight.

The closed-option design characterizes most of the analyzed VPs (15), with only four works based on an open-option design; as for the remaining, one VP implemented both options (NERVE 25|26|37]), whereas the other one can be considered an hybrid between the two designs (Ochs [47]). One explanation for this result is the lower complexity of the closed-option implementation, although some Authors 928 argued that a such an approach may be more suitable for novices who, for example, may still be inexperienced about the procedures to follow in a patient encounter. However, other works reported that many students feel restricted by the closed-option interface [15]26|28|52], preferring either an open-option structure or the possibility to chose between the two variants. It is worth noting that implementing both options allows the use of the same VP in different educational settings. For instance, in NERVE [25 26 [37], the less stressinducing closed-option variant is used in the learning sessions, while rehearsal sessions leverage the less restrictive open-option setting. Another interesting approach is the hybrid model implemented in Ochs 47, where the user can freely interact through voice with the VP. Then, a human facilitator selects, from a set of possible closed-options, the utterance that semantically resembles the original phrase the most, prompting the appropriate response from the patient. The advantage of this approach is that it ease the development burden of what appears to learners as an open-option VP, with the clear disadvantage of preventing its use as a self-learning and self-evaluation tool.

Feedback. With the term feedback we refer to any form of instructional scaffolding enclosed in the simulation itself (i.e., we exclude any feedback external to the simulation, such as post-simulation debrief and reflection sessions with 
mentors and peers). Feedback can be given in many different forms, from explicit messages to discoveries made, questions answered, and visual representations of the current VP state.

While researchers recognize the relevance of immediate and after-action feedback as an essential feature in communication-based VPs (1|29|44|50|53), it is surprising that six of the VPs surveyed (Banszki [453, Maicher [43], Marei [44, Szilas 63, Washburn 66, CESTOLVRClinic [59]) do not embed any type of built-in feedback. The remaining works take different approaches. Four VPs (Dupuy [15, Communicate! [31, VSPR [50/52, Zlotos [70]) offer the possibility, after the simulation is completed, to replay some of its parts and analyze the outcome of different choices. Three simulations (At-Risk in Primary Care [2, Suicide Prevention [48, Schoenthaler [60]) include a virtual instructor, i.e., a virtual tutor that gives advice or feedback based on the user's choices. MPathicVR 2140. employs a multiple session structure, where the first run acts as a learning phase, concluded by an automated and complete feedback provided by the system, whereas the second run (set in the same scenario) serves as an evaluation phase. This type of structure appears to be highly appreciated by students since they can immediately put into practice what they learned during the first phase, taking into account the feedback received. Two VPs (At-Risk in Primary Care [2, Schoenthaler 60]) feature quantitative emotional feedback in the form of an on-screen trust meter that indicates users how effective their communication choices were at building a relation with the patient. Two VPs (Jacklin 28 29, Richardson [54]) offer learners a personalized qualitative feedback at the end of the simulation. CynthiaYoungVP [19] uses an hybrid approach between automated and human feedback. At the end of the simulation, the students can access a web page containing empathy feedback and scores for each response given, where scores are manually assigned by human experts. The approach adopted in NERVE 26 25137] is to inform learners about the number of clinical discoveries and empathic responses available, thus providing inexperienced users with useful guidelines on how to proceed with the conversation. Although it has not been implemented yet, the work in 25] put forth the proposition of providing "cumulative feedback" on how users are developing their skills across multiple patient scenarios. Authors suggest that this feature could both encourage repeated use of the system and act as a motivator for performance improvement. Finally, there is a number of VPs (HOLLIE [1, Dupuy [15, Communicate! [31, MPathic-VR [2140], Schoenthaler [60, UT-Time Portal 6869]) that leverage game elements as feedback. Since the introduction of game elements is a relevant design feature, it is discussed in detail in the following subsection.

Gamification. The idea of introducing game mechanics in any learning experience is to make them more enjoyable and engaging. Researchers and practitioners recognize that game mechanics contribute to making the learning experience more effective, fostering self-improvement and healthy competition between peers 7/16. The mechanics used the most in "gamified" experiences are scores, badges and leaderboards. Scores are a quantitative and immediate form of feedback that acts as an extrinsic motivator to foster users to improve their 
performance. Scoring systems can be found in Dupuy [15, Communicate! [31, MPathic-VR [2140, Schoenthaler 60] and UT-Time Portal 6869. In particular, Communicate! [31] and Schoenthaler 60. provide separate scores for each learning goal (e.g., empathy control, language clarity, and pick up of patient's concerns). Such a feature can help learners to tell the areas in which they are already proficient, distinguishing them from those needing improvement.

Badges are visual representations used in games to prove that the player has reached an intermediate goal on his/her road to mastery. Their purpose is twofold: they are a form of gratification to the learners, and they allow trainees to share achievements with peers and educators. Thus, they also represent an extrinsic motivator for improvement. In our survey, the only simulation we found that implements a badge system is UT-Time Portal 6869. VSPR [50 52 features a system of certificates issued to users at the end of each learning module which shall be regarded as an "intrinsic-only" motivator, since there is no overarching structure that enables users to see each others' achievements.

Finally, leaderboards (or rankings) are a primarily extrinsic motivator that leverage competition with peers when they compare their performance to that of others (it should be noted that, for highly competitive individuals, the act of "climbing the leaderboard" can also be seen as a relevant intrinsic motivator independent of the context). Surprisingly, despite their demonstrated benefits for learning, in our survey, we found no example of public ranking and leaderboards.

A final note is for HOLLIE 1, which implements the very peculiar idea of a Tamagotchi-style VP the learners have to care for (adequately, at regular intervals and in real-time) over two weeks. Here, the leading game mechanics (constant care over a long period) reproduces quite accurately the daily tasks of a nurse leveraging the innate sense of responsibility in the players.

\subsection{Technical features}

This category explores, from a technical perspective, the different solutions that can support (and implement) the choices made in the instructional design, i.e., which are the technical features that enable the accomplishment of the envisioned learning activities. These features include the physical devices required to guarantee the exchange of information between the learner and the system, and the possible communication infrastructure needed to run the simulation.

Presentation format. The surveyed works provide learners with different types of outputs aimed to deliver VP information to the learner and presenting the VP itself. A first rough subdivision is between text-based and graphic representations. In screen-based text simulators, the VP is presented mainly in the form of a collection of text and structured data, with the possible inclusion of images portraying a static patient or exam results. However, the lack of a graphic component capable of displaying a patient that can express emotions as the simulation unfolds (and, consequently, change posture and facial expressions according to its current state) is one of the main limitations of these approaches. Therefore, researchers started extending text-based simulations into learning activities with a relevant graphic component. 
All the VPs surveyed in this work fall in the graphic category, which can be further classified in image, video and 3D. VPs in the image subclass are presented through a series of static images (either photographs or drawings), such as HOLLIE [1] and Marei [44. Some VPs present their case using video, either in the form of live footage (Suicide Prevention [48, VSPR [50|52]) or as a computer-generated offline video (Cynthia Young VP [19]). However, a clear limitation of this approach is its lack of flexibility, since the actor video cannot be re-purposed to portray a different clinical case.

The majority of surveyed simulations fall in the $3 D$ subclass and present the patient and the environment as 3D models rendered in real-time. Their main advantage is that tweaking and expanding a simulation using $3 \mathrm{D}$ characters can be done in a much more modular fashion than with image and video-based VPs. Another advantage is that the sense of immersion and presence are greater than those that can be delivered by image and video-based VPs.

Most of the 3D approaches rely on standard desktop VR (DVR) settings (10, namely AtRiskInPrimaryCare [2, MPathic-VR [21|40], NERVE [25I26]37, Jacklin 28129, Communicate! 31, Richardson [54, Schoenthaler 60, Szilas 63, UTTimePortal 6869, Zlotos 70]). However, since trying to maximize the feeling of immersion and presence is extremely relevant for engaging learners and helping them achieve the expected learning outcomes, some works integrate (partially or fully) immersive technologies. Four of them (Dupuy [15], Banszki 453, Maicher [43, Washburn [66]) take advantage of large volume displays to portray a life-sized and more natural interaction with the patient, and CESTOL VR Clinic [59] uses an HMD for the same purpose. In Ochs [47, three different setups (DVR, immersive VR with an HMD, and immersive VR in a CAVE) are compared to analyze their effect on the sense of presence. The outcome of this experiment demonstrates that immersive environments improve the sense of presence and perception of the VP, with the CAVE scoring slightly better than the HMD. It should be noted, however, that while immersive VR (IVR) offers a higher degree of immersion and presence over DVR, there are still accessibility issues that limit its use, in particular when the VP is intended for self-learning and self-training.

Input Interface. This category describes the input methods through which the user influences the unfolding of the VP simulation. In the case of typed interfaces, user's textual intents are entered by typing on a keyboard or selecting an item in a predefined list of choices. Voice-controlled simulations use natural language, which is then parsed into text through a speech-to-text module, usually offered by external Natural Language Processing (NLP) APIs [1943]. Finally, the integration within the simulation of Natural User Interfaces (NUI) allows to influence the VP state evolution through additional non-verbal input cues such as eye contact, distance, facial expression, gestures and body posture, which can be captured with cameras and other hardware.

Among the analyzed VPs, 14 feature a typed-only input, only five are voicecontrolled (Banszki 4153, Dupuy [15, MPathic-VR 21|40], Ochs 47], CESTOL VR Clinic [59]), Maicher [43] has both options, and Washburn [66] can be con- 
sidered as a hybrid solution since a human facilitator transcribes the spoken commands through a typed interface. One of the reasons behind the limited use of voice controls is the fear, expressed by some Authors [3 477, that NLP systems may be technically hard to implement and prone to wrong transcriptions, which may lead to misunderstandings or unrecognized utterances, break the sense of immersion and cause frustration in the user [8]. This is why some Authors (e.g., Banszki [453, Ochs [47, and Washburn [66]) decided to have a human facilitator taking over the function of the NLP module. Moreover, a VP featuring only voice controls cannot be used by learners with speech impairments 43. However, it should be stressed that, nowadays, speech-to-text APIs have become widely available, and their quality keeps improving; thus, problems related to imprecise transcriptions should be less and less daunting in the coming years. As for the impaired people, a smart solution to achieve maximum flexibility and accessibility could be to let the users choose between typed and voice-controlled interfaces freely. It should also be noted that IVR environments favour the use of voice-controlled interfaces over alternative solutions such as virtual keyboards or situation-specific control boards [59, which are likely to break the sense of immersion and presence and are often cumbersome to use.

Among the analyzed VPs, five of them support also non-verbal input, by either leveraging NUI-based approaches (e.g., using RGBD sensors, like in MPathicVR 2140] and Maicher [43], or standard RGB cameras, like in Dupuy [15]) or having a human controller that observes the user interacting with the VP and updates the VP's response accordingly in terms of gestures and facial expressions (like in Banszki [453]). However, apart from Banszki [453], it appears that this information is largely underutilized to influence the VP's behavior. In Dupuy [15, the users' facial expressions are detected to merely assess their emotional state at the end of the simulation. In Maicher [43, the tracked user position is simply used to adjust the agent's gaze, and there is no specific mention of the way the simulation exploits gestures. Finally, in MPathic-VR 2140, instead of continuously capturing non-verbal communication, learners are forced to assume specific expressions and poses when prompted by the system. In summary, the above discussion highlights that sounder ways of using non-verbal inputs are sorely needed in this particular research field.

Distribution. One relevant technological parameter of the VP simulation is the way the application is distributed (and how learners can access it). In principle, there are two main options. The first option is to deploy the VP as a web-based application that can be accessed over the Internet. Such a simulation often runs inside a web browser (which makes it device-independent), and generally requires a low amount of computational resources. This flexibility can also foster self-learning (since simulations can be accessed at places and times convenient for the learner) and helps reduce costs (since learning can be carried out online). However, since web-based applications are required to be portable on many devices (including mobile ones), they generally sacrifice technical characteristics and computational complexity in favour of accessibility. On the other hand, standalone applications are deployed locally on a computer or workstation. 
These simulations can implement more advanced and complex features since they can leverage the full computational power of a dedicated machine, and integrate external devices or sensors (such as high-quality cameras and microphones).

In our survey, we found a total of ten web-based and eight standalone applications; in two cases, this information was undisclosed in the paper, whereas in one case (Maicher [43]), the VP was deployed in both variants. This latter work is interesting since it shows how a standalone version can trade off some of the flexibility of the web one with a broad array of features (such as voice control and gesture/posture detection). The Authors observed that students were significantly more engaged with the standalone VP, whereas in the web version they had to focus on typing and reading, which make them be less prone to notice the subtle non-verbal cues manifested by the patient.

Nonetheless, it should be stressed that technology is advancing rapidly, and personal devices come equipped with ever better microphones, cameras and computational power, which can reduce the technological gap between (desktop-only) standalone applications and web-based ones. Further discussions on this topic are included in Section 4 .

\subsection{Effectiveness of design elements}

The general effectiveness of VPs on developing communication skills has been discussed by several Authors 41/51/55]. A common complaint in VP-related literature is the lack of a standardized terminology that, coupled with a considerable heterogeneity in study design, makes the retrieval and evaluation of relevant works a troublesome task. Despite this situation, both [41] and [51] concluded that, when appropriately contextualized in a well thought out educational context, VPs are indeed useful for developing, practising and building confidence about communication and other skills like, e.g., decision making and teamwork.

Based on these findings, one possible question arising from our review is if the surveyed papers provide pieces of evidence about the effects on learning outcomes and efficacy of the simulation of the different instructional design elements and the technical features available. Unfortunately, the answer is negative. In most of the analyzed works, the Authors reported only users' feedback or comments about a particular element/feature, and a direct comparison between different design choices is missing. The only notable exceptions are three. The first one is represented by [47, in which different presentation formats were assessed, showing that immersive VR technologies yield superior results when compared to non-immersive ones. The second one concerns the distribution method 43]. The Authors found that a standalone application can provide a considerably higher level of engagement than its web-based counterpart thanks to the possibility to leverage advanced technical features (voice-controlled input and large volume displays) to increase immersion and focus on the task at hand. The third one compared closed and open-option unfolding designs, highlighting the advantages and disadvantages of each variant [26]. 


\section{Open areas of research}

The surveyed papers show that, despite exciting results obtained, fully understanding how to develop effective VPs for patient-doctor communication training requires further work. Reasons are related to the fact that either the technological components have not been fully explored yet or results are still inadequate to fully assess the effectiveness of different design approaches. Thus, in this section, we briefly discuss some open problems and present areas requiring further research.

Assessment of design elements. As discussed in Section 3.3 the current literature lacks a thorough evaluation of the effectiveness of alternative designs. This observation highlights the fact that further work has to be done to develop a better understanding of instructional elements and technical features that VP simulations can offer in order to achieve the desired learning outcomes.

Scope. Another comment can be made on the specific communication learning context. While several core skill domains jointly contribute to a patient's health and satisfaction (like relationship building, information gathering, patient education, shared decision making and breaking bad news [56]), most of the surveyed VP simulations focus only on one specific domain. This observation highlights the need to develop novel approaches capable of addressing simultaneously the multiple communication challenges one has to face when interacting with a real patient, thus helping to improve the overall learner's communication skills.

Authoring tools. Implementing VPs is a cumbersome and complicated process, which requires taking into account several different elements (NLP, emotion modelling, affective computing, 3D animations, etc.), which, in turn, involve specific technological and technical skills. Usually, the development of a VP is a cyclical process of research, refinement and validation with experts that can take a considerable amount of time 58 . Thus, there is the need to develop simple (and effective) authoring tools that can allow developers to support clinical educators in the rapid design, prototyping and deploying of VPs in a variety of use cases. Examples of authoring tools for narrative-style VPs with 3D graphics are very scarce in the literature. The work presented in 31] integrates a scenario builder that allows clinical educators to design the unfolding of their cases. This authoring tool exploits a domain reasoner where the response of the virtual agent is determined not only by the previous dialogue that the user chose, but also by other parameters like the agent's current emotional state. However, this tool lacks the possibility to customize the virtual environment or the VP's aesthetics. The NERVE VP [25 26/37] is built upon the Virtual People Factory [58, a web application that enables the users to build conversational models using an un-annotated corpus retrieval approach based on keyword matching. Another interesting example is SIDNIE (Scaffolded Interviews Developed by Nurses in Education [14). This tool allows clinical educators to edit the patient's medical status, dialogue options and physical appearance. However, to our knowledge, SIDNIE has not been deployed in any publicly available form, and appears to be aimed exclusively at nurse training scenarios. 
In other application areas (such as building clinical skills and problem-solving abilities), the extensive use of tools such as DecisionSim, OpenLabyrinth and Web-SP 13 is a clear demonstration of the fact that an easy-to-use authoring tool is a determinant factor for the success of a VP application. However, compared to these areas, the specific context of patient-doctor communication training involves more complex systems, with $3 \mathrm{D}$ visuals and branched narratives offering a more realistic interaction, which makes the development of authoring tools in this area much more challenging 64 .

Emerging web technologies. In the previous sections, we highlighted that personal devices are coming with better and better hardware and computational power, thus helping to narrow the gap between standalone and web-based applications. Another contribution will inevitably come from recent advances in web-based technology, like, e.g., WebXR 1 . WebXR is a device-independent framework that allows users to develop and share VR and AR applications over the Internet, with considerable support for different hardware and web browsers. In addition, game-streaming platforms such as Google Stadid 2 are a very promising workaround for the limited computational capabilities of personal devices. With these platforms, the bulk of the computation is processed on the server side, then the pre-rendered output is streamed to the final user's device. The implementation of such technological solutions in the immediate future will enable the applications to combine the accessibility of current web-based software with the computational complexity of standalone applications run on a dedicated machine.

Multiple virtual humans. Interacting with a relative or another health care provider are considered crucial aspects of a clinician's communication skills 23134. However, VP simulations usually include only two actors: the learner (possibly represented by an avatar) and a unique Non-Playable Character (NPC), i.e, a virtual human not controlled by the trainee that represents the patient. The only two examples that include more than one NPC besides the patient are the Medical Interview Episode of the UTTimePortal 6869 (which incorporates a patient and a caregiver), and MPathic-VR 21140] (which includes a patient's relative and a nurse). Beyond this observation, we should also note that another interesting future development (still untouched in the field of VPs for patient-doctor communication skills, to the best of our knowledge) could be to provide the possibility of interacting (within the simulation) with other humancontrolled avatars, in a way similar to that proposed by approaches focused on inter-professional communication in emergency medical situations [3].

Immersive VR and AR. There is a general understanding among researchers that increasing the level of immersion and realism of the simulations (e.g., using large volume displays, HMDs, spatialized 3D audio, higher fidelity graphics and animations) leads to more believable human-computer interactions [10]32, which in turns help improve the users' communication and empathic skills [47/67] and, ultimatley, the learning outcomes in general [42]. However,

1 https://www.w3.org/TR/webxr/

2 https://stadia.google.com/ 
surprisingly, the use of IVR technologies in this specific context appears to be quite limited. Only two VPs out of 21, i.e., Ochs [4] and CESTOL VR Clinic [59], mention the use of IVR, and AR appears to be completely unexplored. The primary obstacles to the adoption of IVR or AR in VP simulations seem to be the complexity, challenges and costs of development steps 67.

Fortunately, things are going to change rapidly. In recent years, the availability and quality of VR devices have increased considerably, and their cost has decreased dramatically. These factors contribute (together with the availability of high-end development platforms such as Unity or Unreal engine) to reducing overall costs and efforts for developing IVR and AR applications. Furthermore, IVR offers currently a truly immersive, unbroken environment that can shift the cognitive load directed on imagining oneself "being there" in VR towards solving the task at hand. In turn, higher immersion and visual fidelity can have positive effects on learning [11, [27. Thus, we expect that, soon, VR and AR will contribute to improving the state of the art in this research field.

Fully-fledged non-verbal input. In our opinion, this is a major lack in current designs. The unfolding of the simulation's narrative should be dictated (in tandem) by both user's verbal and non-verbal behaviours. To this end, developers of future VPs should attempt to fully leverage non-verbal cues as a factor that actively influences the state of the agent. For instance, the same utterance should have a different outcome if the user maintains eye contact with the patient, looks in another direction, and is fidgeting or exhibiting an incoherent facial expression. The extraction of para-linguistic factors such as tone of voice, loudness, inflection, rhythm, and pitch can provide information about the actual emotional states of the other peer in the communication. Prosody must be addressed with great attention since it is one of the main ways to express empathy and can have a considerable impact in increasing patient satisfaction [34. Thus, computational mechanisms capable of extracting these variables from the analysis of the user's voice are sorely needed. The same para-linguistic factors should be also available to modulate the VP response according to its emotional states. In fact, one of the problems with present text-to-speech libraries is that they pronounce everything with the same tone, which makes it impossible to communicate feelings through voice.

\section{Conclusion}

In our research we found many different examples of VPs focused on providerpatient communication and various approaches to their design. However, we feel that there is not a single VP that realizes the full potential of this learning tool. Some research areas still need to be explored further. The broad range of educational use cases in healthcare suggests that VP applications should be as modular and adaptable as possible. Effective and user-friendly authoring tools are very rarely implemented while being, in our opinion, a crucial feature to ensure the adoption of a VP simulation by clinical educators. The use of technologies such as VR, AR, and advanced NLP also needs to be explored more 
in depth, as they may give VP simulations the edge they need to be effectively used in daily practice. We also feel that recent developments in web-based technologies will also reduce those compromises between accessibility and advanced technical possibilities that today are still required in many situations.

\section{References}

1. Adefila, A., Opie, J., Ball, S., Bluteau, P.: Students' engagement and learning experiences using virtual patient simulation in a computer supported collaborative learning environment. Innovations in Education and Teaching International 57(1), 50-61 (2020)

2. Albright, G., Bryan, C., Adam, C., McMillan, J., Shockley, K.: Using virtual patient simulations to prepare primary health care professionals to conduct substance use and mental health screening and brief intervention. Journal of the American Psychiatric Nurses Association 24(3), 247-259 (2018)

3. Anbro, S.J., Szarko, A.J., Houmanfar, R.A., Maraccini, A.M., Crosswell, L.H., Harris, F.C., Rebaleati, M., Starmer, L.: Using virtual simulations to assess situational awareness and communication in medical and nursing education: A technical feasibility study. Journal of Organizational Behavior Management pp. 1-11 (2020)

4. Bánszki, F., Beilby, J., Quail, M., Allen, P., Brundage, S., Spitalnick, J.: A clinical educator's experience using a virtual patient to teach communication and interpersonal skills. Australasian Journal of Educational Technology 34(3) (2018)

5. Bearman, M., Cesnik, B., Liddell, M.: Random comparison of 'virtual patient'models in the context of teaching clinical communication skills. Medical education 35(9), 824-832 (2001)

6. Bearman, M., Palermo, C., Allen, L.M., Williams, B.: Learning empathy through simulation: a systematic literature review. Simulation in healthcare 10(5), 308-319 (2015)

7. Benedict, N., Schonder, K., McGee, J.: Promotion of self-directed learning using virtual patient cases. American Journal of Pharmaceutical Education 77(7) (2013)

8. Bloodworth, T., Cairco, L., McClendon, J., Hodges, L.F., Babu, S., Meehan, N.K., Johnson, A., Ulinski, A.C.: Initial evaluation of a virtual pediatric patient system (2010)

9. Carnell, S., Halan, S., Crary, M., Madhavan, A., Lok, B.: Adapting virtual patient interviews for interviewing skills training of novice healthcare students. In: International Conference on Intelligent Virtual Agents. pp. 50-59. Springer (2015)

10. Chuah, J.H., Robb, A., White, C., Wendling, A., Lampotang, S., Kopper, R., Lok, B.: Exploring agent physicality and social presence for medical team training. Presence: Teleoperators and Virtual Environments 22(2), 141-170 (2013)

11. Coulter, R., Saland, L., Caudell, T., Goldsmith, T.E., Alverson, D.: The effect of degree of immersion upon learning performance in virtual reality simulations for medical education. InMedicine Meets Virtual Reality 15, 155 (2007)

12. Dibbelt, S., Schaidhammer, M., Fleischer, C., Greitemann, B.: Patient-doctor interaction in rehabilitation: is there a relationship between perceived interaction quality and long term treatment results? Die Rehabilitation 49(5), 315-325 (2010)

13. Doloca, A., ŢĂNCUlESCU, O., Ciongradi, I., Trandafir, L., Stoleriu, S., Ifteni, G.: Comparative study of virtual patient applications. Proc. of the Romanian Academy, Series A 16(3), 466-473 (2015) 
14. Dukes, L.C., Meehan, N., Hodges, L.F.: Participatory design of a pediatric virtual patient creation tool. In: 2016 IEEE International Conference on Healthcare Informatics (ICHI). pp. 449-455. IEEE (2016)

15. Dupuy, L., Micoulaud-Franchi, J.A., Cassoudesalle, H., Ballot, O., Dehail, P., Aouizerate, B., Cuny, E., de Sevin, E., Philip, P.: Evaluation of a virtual agent to train medical students conducting psychiatric interviews for diagnosing major depressive disorders. Journal of Affective Disorders 263, 1-8 (2020)

16. Festinger, L.: A theory of social comparison processes. Human relations $\mathbf{7}(2), 117$ 140 (1954)

17. Fiscella, K., Meldrum, S., Franks, P., Shields, C.G., Duberstein, P., McDaniel, S.H., Epstein, R.M.: Patient trust: is it related to patient-centered behavior of primary care physicians? Medical care pp. 1049-1055 (2004)

18. Forrest, K., McKimm, J., Edgar, S.: Essential simulation in clinical education. John Wiley \& Sons (2013)

19. Foster, A., Chaudhary, N., Kim, T., Waller, J.L., Wong, J., Borish, M., Cordar, A., Lok, B., Buckley, P.F.: Using virtual patients to teach empathy: a randomized controlled study to enhance medical students' empathic communication. Simulation in Healthcare 11(3), 181-189 (2016)

20. Franks, P., Fiscella, K., Shields, C.G., Meldrum, S.C., Duberstein, P., Jerant, A.F., Tancredi, D.J., Epstein, R.M.: Are patients' ratings of their physicians related to health outcomes? The Annals of Family Medicine 3(3), 229-234 (2005)

21. Guetterman, T.C., Sakakibara, R., Baireddy, S., Kron, F.W., Scerbo, M.W., Cleary, J.F., Fetters, M.D.: Medical students' experiences and outcomes using a virtual human simulation to improve communication skills: Mixed methods study. Journal of medical Internet research 21(11), e15459 (2019)

22. Ha, J.F., Longnecker, N.: Doctor-patient communication: a review. Ochsner J 10(1), 38-43 (2010)

23. Hallin, K., Henriksson, P., Dalén, N., Kiessling, A.: Effects of interprofessional education on patient perceived quality of care. Medical Teacher 33(1), e22-e26 (2011)

24. Hickson, G.B., Federspiel, C.F., Pichert, J.W., Miller, C.S., Gauld-Jaeger, J., Bost, P.: Patient complaints and malpractice risk. Jama 287(22), 2951-2957 (2002)

25. Hirumi, A., Johnson, T., Reyes, R.J., Lok, B., Johnsen, K., Rivera-Gutierrez, D.J., Bogert, K., Kubovec, S., Eakins, M., Kleinsmith, A., et al.: Advancing virtual patient simulations through design research and interplay: part ii-integration and field test. Educational technology research and development 64(6), 1301-1335 (2016)

26. Hirumi, A., Kleinsmith, A., Johnsen, K., Kubovec, S., Eakins, M., Bogert, K., Rivera-Gutierrez, D.J., Reyes, R.J., Lok, B., Cendan, J.: Advancing virtual patient simulations through design research and interplay: part i: design and development. Educational Technology Research and Development 64(4), 763-785 (2016)

27. Huerta, R.: Measuring the impact of narrative on player's presence and immersion in a first person game environment. The University of Texas-Pan American (2012)

28. Jacklin, S., Chapman, S., Maskrey, N.: Virtual patient educational intervention for the development of shared decision-making skills: a pilot study. BMJ Simulation and Technology Enhanced Learning 5(4), 215-217 (2019)

29. Jacklin, S., Maskrey, N., Chapman, S.: Improving shared decision making between patients and clinicians: Design and development of a virtual patient simulation tool. JMIR medical education 4(2), e10088 (2018) 
30. Janda, M.S., Mattheos, N., Nattestad, A., Wagner, A., Nebel, D., Färbom, C., Lê, D.H., Attström, R.: Simulation of patient encounters using a virtual patient in periodontology instruction of dental students: design, usability, and learning effect in history-taking skills. European Journal of Dental Education 8(3), 111-119 (2004)

31. Jeuring, J., Grosfeld, F., Heeren, B., Hulsbergen, M., IJntema, R., Jonker, V., Mastenbroek, N., van der Smagt, M., Wijmans, F., Wolters, M., et al.: Communicate! - a serious game for communication skills - . In: Design for teaching and learning in a networked world, pp. 513-517. Springer (2015)

32. Johnsen, K., Lok, B.: An evaluation of immersive displays for virtual human experiences. In: 2008 IEEE virtual reality conference. pp. 133-136. IEEE (2008)

33. Judge, T.A., Ilies, R.: Affect and job satisfaction: a study of their relationship at work and at home. Journal of applied psychology 89(4), 661 (2004)

34. Kee, J.W., Khoo, H.S., Lim, I., Koh, M.Y.: Communication skills in patient-doctor interactions: learning from patient complaints. Health Professions Education 4(2), 97-106 (2018)

35. Kelley, J.M., Lembo, A.J., Ablon, J.S., Villanueva, J.J., Conboy, L.A., Levy, R., Marci, C.D., Kerr, C., Kirsch, I., Jacobson, E.E., et al.: Patient and practitioner influences on the placebo effect in irritable bowel syndrome. Psychosomatic medicine 71(7), 789 (2009)

36. King, A., Hoppe, R.B.: "best practice" for patient-centered communication: A narrative review. Journal of Graduate Medical Education 5(3), 385-393 (Sep 2013). https://doi.org/10.4300/jgme-d-13-00072.1, https://doi.org/10.4300/jgme-d-13-00072.1

37. Kleinsmith, A., Rivera-Gutierrez, D., Finney, G., Cendan, J., Lok, B.: Understanding empathy training with virtual patients. Computers in human behavior 52, 151-158 (2015)

38. Kneebone, R., Nestel, D., Wetzel, C., Black, S., Jacklin, R., Aggarwal, R., Yadollahi, F., Wolfe, J., Vincent, C., Darzi, A.: The human face of simulation: patientfocused simulation training. Academic Medicine 81(10), 919-924 (2006)

39. Kohatsu, N.D., Gould, D., Ross, L.K., Fox, P.J.: Characteristics associated with physician discipline: a case-control study. Archives of internal medicine 164(6), 653-658 (2004)

40. Kron, F.W., Fetters, M.D., Scerbo, M.W., White, C.B., Lypson, M.L., Padilla, M.A., Gliva-McConvey, G.A., Belfore II, L.A., West, T., Wallace, A.M., et al.: Using a computer simulation for teaching communication skills: A blinded multisite mixed methods randomized controlled trial. Patient education and counseling 100(4), 748-759 (2017)

41. Lee, J., Kim, H., Kim, K.H., Jung, D., Jowsey, T., Webster, C.: Effective virtual patient simulators for medical communication training: a systematic review. Medical Education (2020)

42. Limniou, M., Roberts, D., Papadopoulos, N.: Full immersive virtual environment cavetm in chemistry education. Computers \& Education 51(2), 584-593 (2008)

43. Maicher, K., Danforth, D., Price, A., Zimmerman, L., Wilcox, B., Liston, B., Cronau, H., Belknap, L., Ledford, C., Way, D., et al.: Developing a conversational virtual standardized patient to enable students to practice history-taking skills. Simulation in Healthcare 12(2), 124-131 (2017)

44. Marei, H., Al-Eraky, M., Almasoud, N., Donkers, J., Van Merrienboer, J.: The use of virtual patient scenarios as a vehicle for teaching professionalism. European Journal of Dental Education 22(2), e253-e260 (2018) 
45. McCoy, L., Pettit, R.K., Lewis, J.H., Allgood, J.A., Bay, C., Schwartz, F.N.: Evaluating medical student engagement during virtual patient simulations: a sequential, mixed methods study. BMC medical education 16(1), 20 (2016)

46. Nestel, D., Tabak, D., Tierney, T., Layat-Burn, C., Robb, A., Clark, S., Morrison, T., Jones, N., Ellis, R., Smith, C., McNaughton, N., Knickle, K., Higham, J., Kneebone, R.: Key challenges in simulated patient programs: An international comparative case study. BMC Medical Education 11(1) (Sep 2011). https://doi.org/10.1186/1472-6920-11-69, https://doi.org/10.1186/1472-6920-11-69

47. Ochs, M., Mestre, D., De Montcheuil, G., Pergandi, J.M., Saubesty, J., Lombardo, E., Francon, D., Blache, P.: Training doctors' social skills to break bad news: evaluation of the impact of virtual environment displays on the sense of presence. Journal on Multimodal User Interfaces 13(1), 41-51 (2019)

48. O’Brien, K.H.M., Fuxman, S., Humm, L., Tirone, N., Pires, W.J., Cole, A., Grumet, J.G.: Suicide risk assessment training using an online virtual patient simulation. Mhealth 5 (2019)

49. Papadakis, M.A., Teherani, A., Banach, M.A., Knettler, T.R., Rattner, S.L., Stern, D.T., Veloski, J.J., Hodgson, C.S.: Disciplinary action by medical boards and prior behavior in medical school. New England Journal of Medicine 353(25), 2673-2682 (2005)

50. Peddle, M., Bearman, M., Mckenna, L., Nestel, D.: Exploring undergraduate nursing student interactions with virtual patients to develop 'non-technical skills' through case study methodology. Advances in Simulation 4(1), 2 (2019)

51. Peddle, M., Bearman, M., Nestel, D.: Virtual patients and nontechnical skills in undergraduate health professional education: an integrative review. Clinical Simulation in Nursing 12(9), 400-410 (2016)

52. Peddle, M., Mckenna, L., Bearman, M., Nestel, D.: Development of non-technical skills through virtual patients for undergraduate nursing students: an exploratory study. Nurse education today 73, 94-101 (2019)

53. Quail, M., Brundage, S.B., Spitalnick, J., Allen, P.J., Beilby, J.: Student selfreported communication skills, knowledge and confidence across standardised patient, virtual and traditional clinical learning environments. BMC medical education 16(1), 73 (2016)

54. Richardson, C.L., Chapman, S., White, S.: Virtual patient educational programme to teach counselling to clinical pharmacists: development and proof of concept. BMJ Simulation and Technology Enhanced Learning 5(3), 167-169 (2019)

55. Richardson, C.L., White, S., Chapman, S.: Virtual patient technology to educate pharmacists and pharmacy students on patient communication: a systematic review. BMJ Simulation and Technology Enhanced Learning (2019)

56. Riedl, D., Schüßler, G.: The influence of doctor-patient communication on health outcomes: a systematic review. Zeitschrift für Psychosomatische Medizin und Psychotherapie 63(2), 131-150 (2017)

57. Rogers, L.: Developing simulations in multi-user virtual environments to enhance healthcare education. British Journal of Educational Technology 42(4), 608-615 (2011)

58. Rossen, B., Lind, S., Lok, B.: Human-centered distributed conversational modeling: Efficient modeling of robust virtual human conversations. In: International Workshop on Intelligent Virtual Agents. pp. 474-481. Springer (2009)

59. Sapkaroski, D., Baird, M., McInerney, J., Dimmock, M.R.: The implementation of a haptic feedback virtual reality simulation clinic with dynamic patient interaction 
and communication for medical imaging students. Journal of medical radiation sciences 65(3), 218-225 (2018)

60. Schoenthaler, A., Albright, G., Hibbard, J., Goldman, R.: Simulated conversations with virtual humans to improve patient-provider communication and reduce unnecessary prescriptions for antibiotics: a repeated measure pilot study. JMIR medical education 3(1), e7 (2017)

61. Stelfox, H.T., Gandhi, T.K., Orav, E.J., Gustafson, M.L.: The relation of patient satisfaction with complaints against physicians and malpractice lawsuits. The American journal of medicine 118(10), 1126-1133 (2005)

62. Stewart, A.M.: Effective physician-patient communication and health outcomes: a review. CMAJ : Canadian Medical Association journal = journal de l'Association medicale canadienne pp. 1423-1433 (1995)

63. Szilas, N., Chauveau, L., Andkjaer, K., Luiu, A.L., Bétrancourt, M., Ehrler, F.: Virtual patient interaction via communicative acts. In: Proceedings of the 19th ACM International Conference on Intelligent Virtual Agents. pp. 91-93 (2019)

64. Talbot, T.B., Sagae, K., John, B., Rizzo, A.A.: Sorting out the virtual patient: how to exploit artificial intelligence, game technology and sound educational practices to create engaging role-playing simulations. International Journal of Gaming and Computer-Mediated Simulations (IJGCMS) 4(3), 1-19 (2012)

65. Urresti-Gundlach, M., Tolks, D., Kiessling, C., Wagner-Menghin, M., Härtl, A., Hege, I.: Do virtual patients prepare medical students for the real world? development and application of a framework to compare a virtual patient collection with population data. BMC medical education 17(1), 174 (2017)

66. Washburn, M., Parrish, D.E., Bordnick, P.S.: Virtual patient simulations for brief assessment of mental health disorders in integrated care settings. Social Work in Mental Health 18(2), 121-148 (2020)

67. Zielke, M.A., Zakhidov, D., Hardee, G., Evans, L., Lenox, S., Orr, N., Fino, D., Mathialagan, G.: Developing virtual patients with vr/ar for a natural user interface in medical teaching. In: 2017 IEEE 5th International Conference on Serious Games and Applications for Health (SeGAH). pp. 1-8. IEEE (2017)

68. Zielke, M.A., Zakhidov, D., Jacob, D., Hardee, G.: Beyond fun and games: toward an adaptive and emergent learning platform for pre-med students with the ut time portal. In: 2016 IEEE International Conference on Serious Games and Applications for Health (SeGAH). pp. 1-8. IEEE (2016)

69. Zielke, M.A., Zakhidov, D., Jacob, D., Lenox, S.: Using qualitative data analysis to measure user experience in a serious game for premed students. In: International Conference on Virtual, Augmented and Mixed Reality. pp. 92-103. Springer (2016)

70. Zlotos, L., Power, A., Hill, D., Chapman, P.: A scenario-based virtual patient program to support substance misuse education. American journal of pharmaceutical education $\mathbf{8 0}(3)$ (2016) 